\title{
Emission of Dioxin-like Compounds and Flame Retardants from Commercial Facilities Handling Deca-BDE and Their Downstream Sewage Treatment Plants
}

Go Suzuki, ${ }^{* 1}$ Hidenori Matsukami, ${ }^{1}$ Chieko Michinaka, ${ }^{1}$ Shunji Hashimoto, ${ }^{2}$ Kei Nakayama, ${ }^{3}$ Shin-ichi Sakai ${ }^{4}$

${ }^{1}$ Center for Material Cycles and Waste Management Research, National Institute for Environmental Studies, Tsukuba 305-8506, Japan

${ }^{2}$ Center for Environmental Measurement and Analysis, National Institute for Environmental Studies, Tsukuba 305-8506, Japan

${ }^{3}$ Center for Marine Environmental Studies, Ehime University, 2-5 Bunkyo-cho, Matsuyama 790-8577, Japan

${ }^{4}$ Environment Preservation Research Center, Kyoto University, Kyoto 606-8501, Japan

*Corresponding author: Go Suzuki, Ph.D.

Phone: +81-29-850-2205

E-mail: g-suzuki@nies.go.jp

Number of pages: 21 


\section{Experimental section}

Table S1 Basic information for the 16 Deca-BDE handling facilities investigated in the present study

\begin{tabular}{|c|c|c|c|c|c|c|}
\hline Facility ID & & Industry & Main product & $\begin{array}{l}\text { FY2015 PRTR } \\
\text { Deca-BDE (kg) }\end{array}$ & $\begin{array}{l}\text { Daily amount of } \\
\text { effluent }\left(\mathrm{m}^{3}\right)\end{array}$ & $\begin{array}{l}\text { Destination of } \\
\text { effluent }\left(\mathrm{m}^{3}\right)\end{array}$ \\
\hline Deca-BDE handling facility-1 & DHF-1 & Rubber manufacturing & Industrial rubber & 2.6 & $<50$ & River \\
\hline Deca-BDE handling facility-2 & DHF-2 & Plastic manufacturing & Industrial plastic & 1,400 & $<50$ & STP \\
\hline Deca-BDE handling facility-3 & DHF-3 & Chemical & Plastic additive & 20 & 0.4 & STP \\
\hline Deca-BDE handling facility-4 & DHF-4 & Chemical & Adhesive for industry & 150 & 60 & STP \\
\hline Deca-BDE handling facility-5 & DHF-5 & Non-ferrous metal manufacturing & Electronic wire & 410 & 940 & STP \\
\hline Deca-BDE handling facility- 6 & DHF-6 & Textile & Dyed textile & 0 & $<50$ & STP \\
\hline Deca-BDE handling facility-7 & DHF-7 & Non-ferrous metal manufacturing & Electronic wire & 3,400 & 155 & River \\
\hline Deca-BDE handling facility- 8 & DHF-8 & Textile & Fabric, building material & 23 & 25,000 & Sea \\
\hline Deca-BDE handling facility-9 & DHF-9 & Textile & Car sheet, fabric & 130 & 1,350 & STP \\
\hline Deca-BDE handling facility-10 & DHF-10 & Textile & Car sheet, fabric & 280 & 1,100 & River \\
\hline Deca-BDE handling facility-11 & DHF-11 & Textile & Car sheet, fabric & 640 & $<50$ & River \\
\hline Deca-BDE handling facility-12 & DHF-12 & Textile & Car sheet, fabric & 73,000 & $<50$ & River \\
\hline Deca-BDE handling facility-13 & DHF-13 & Textile & Banner, flag & 300 & Unknown & STP \\
\hline Deca-BDE handling facility-14 & DHF-14 & Rubber manufacturing & Tire & 53 & Unknown & STP \\
\hline Deca-BDE handling facility-15 & DHF-15 & Non-ferrous metal manufacturing & Electronic wire & 5.6 & Unknown & STP \\
\hline Deca-BDE handling facility-16 & DHF-16 & Non-ferrous metal manufacturing & Electronic wire & 260 & Unknown & STP \\
\hline
\end{tabular}

PRTR: Pollutant Release and Transfer Register, STP: sewage treatment plant 
Table S2 Basic information for the three sewage treatment plants investigated in the present study

\begin{tabular}{|c|c|c|c|c|c|c|c|}
\hline Plant ID & & $\begin{array}{l}\text { Collecting } \\
\text { area }\left(\mathrm{km}^{2}\right)\end{array}$ & $\begin{array}{l}\text { Collecting } \\
\text { population }\end{array}$ & Sewer system & $\begin{array}{c}\text { Residence time } \\
\text { (h) }\end{array}$ & $\begin{array}{l}\text { Daily amount } \\
\text { effluent }\left(\mathrm{m}^{3}\right)\end{array}$ & $\begin{array}{c}\text { Destination of } \\
\text { effluent }\end{array}$ \\
\hline Sewage treatment plant-1 & STP-1 & 126.25 & 542,400 & Separate & $16-20$ & 169,373 & Sea \\
\hline Sewage treatment plant-2 & STP-2 & 118.69 & 576,500 & Separate/combined & 20 & 322,800 & River \\
\hline Sewage treatment plant- 3 & STP-3 & 12.37 & 58,000 & Separate/combined & $16-20$ & 264,100 & River \\
\hline
\end{tabular}

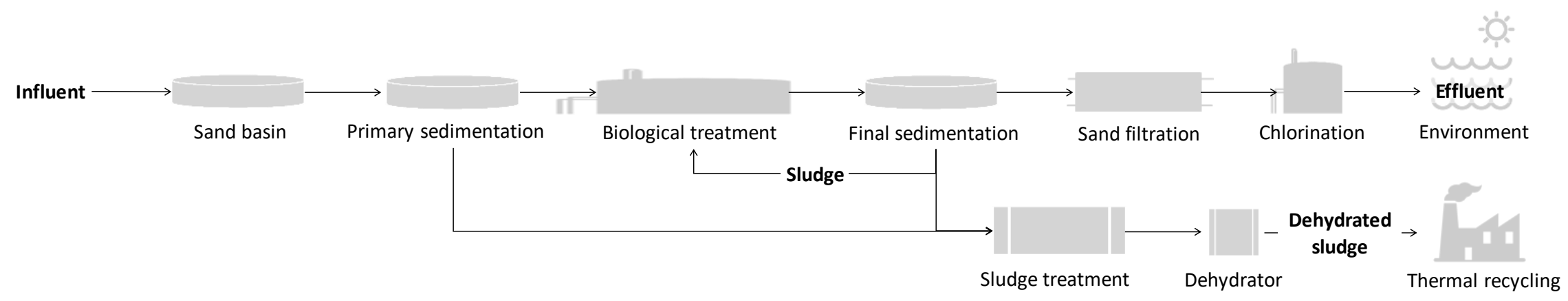

Figure S1 Flow of influent through the sewage treatment plants

In the STP-3, the sludge sedimented in the final sedimentation basin was returned to the biological treatment tank as return sludge and a part of the sludge is thermally recycled after dehydration as excess sludge. 
Table S3 Sampling date, weather, water temperature, $\mathrm{pH}$, electrical conductivity (EC), and concentrations of suspended solids (SS) for samples from the deca-BDE handling facilities

\begin{tabular}{|c|c|c|c|c|c|c|c|}
\hline Sample ID & Samples & $\begin{array}{c}\text { Sampling } \\
\text { date }\end{array}$ & Weather & $\begin{array}{c}\text { Water } \\
\text { Temp. }\left({ }^{\circ} \mathrm{C}\right)\end{array}$ & $\mathrm{pH}$ & $\begin{array}{c}\mathrm{EC} \\
(\mathrm{mS} / \mathrm{m})\end{array}$ & $\begin{array}{c}\mathrm{SS} \\
(\mathrm{mg} / \mathrm{L})\end{array}$ \\
\hline DHF-1 & Effluent & Feb 2018 & Sunny & 21.6 & 7.7 & 25.6 & 1.3 \\
\hline DHF-2 & Effluent & Feb 2018 & Sunny & 11.0 & 7.6 & 182.2 & 4.5 \\
\hline DHF-3 & Effluent & Feb 2018 & Cloudy & 10.3 & 9.2 & 98.4 & 79 \\
\hline DHF-5 & Effluent & Dec 2017 & Sunny & 16.9 & 7.1 & 41.1 & 14 \\
\hline DHF-6 & Effluent & Dec 2017 & Sunny & 15.0 & 7.0 & 51.6 & 53 \\
\hline DHF-7 & Effluent & Feb 2018 & Cloudy & 14.4 & 7.6 & 33.1 & 0.33 \\
\hline \multirow{2}{*}{ DHF-8 } & Effluent-1 & Dec 2017 & Wet & 18.0 & 7.2 & 163.9 & 550 \\
\hline & Effluent-2 & Oct 2019 & Cloudy & 30.5 & 7.3 & 185 & 23 \\
\hline DHF-9 & Effluent-2 & Oct 2019 & Cloudy & 28.1 & 7.5 & 115.9 & 92 \\
\hline \multirow{2}{*}{ DHF-10 } & Effluent-1 & Dec 2017 & Wet & 21.1 & 7.0 & 135 & 93 \\
\hline & Effluent-2 & Oct 2019 & Cloudy & 35.5 & 7.0 & 33.7 & 34 \\
\hline DHF-11 & Effluent & Feb 2018 & Sunny & 18.1 & 7.2 & 78.1 & 9.2 \\
\hline DHF-12 & Effluent & Feb 2018 & Sunny & 15.9 & 8.5 & 26.5 & 0.088 \\
\hline
\end{tabular}

DHF: deca-BDE handling facility, NA: Not available. 
Table S4 Sampling date, weather, water temperature, $\mathrm{pH}$, electrical conductivity (EC), and concentrations of suspended solids (SS) for samples from the sewage treatment plants

\begin{tabular}{|c|c|c|c|c|c|c|c|c|}
\hline Sample ID & Samples & $\begin{array}{l}\text { Sampling } \\
\text { date }\end{array}$ & Weather & $\begin{array}{c}\text { Water } \\
\text { Temp. }\left({ }^{\circ} \mathrm{C}\right)\end{array}$ & $\mathrm{pH}$ & $\begin{array}{c}\mathrm{EC} \\
(\mathrm{mS} / \mathrm{m})\end{array}$ & $\begin{array}{c}\mathrm{SS} \\
(\mathrm{mg} / \mathrm{L})\end{array}$ & Note \\
\hline \multirow{2}{*}{ STP-1 } & Influent & \multirow{2}{*}{ Dec 2017} & \multirow{2}{*}{ Cloudy } & 17.8 & 6.5 & 199.3 & 150 & \multirow{2}{*}{$\begin{array}{l}\text { Receiving DHF-13 } \\
\text { effluent }\end{array}$} \\
\hline & Effluent & & & 9.9 & 6.7 & 170.2 & 0.06 & \\
\hline \multirow{2}{*}{ STP-2 } & Influent & \multirow{2}{*}{ Feb 2018} & \multirow{2}{*}{ Sunny } & 19.3 & 7.6 & 94.2 & 56 & \multirow{2}{*}{$\begin{array}{l}\text { Receiving DHF-14 to - } \\
16 \text { effluent }\end{array}$} \\
\hline & Effluent & & & 19.5 & 6.7 & 66.0 & 1.4 & \\
\hline \multirow{7}{*}{ STP-3 } & Influent & & & 24.6 & 7.3 & 50.1 & 70 & \multirow{7}{*}{$\begin{array}{l}\text { Receiving DHF-9 } \\
\text { effluent }\end{array}$} \\
\hline & PS effluent & & & 26.0 & 7.2 & 491 & 23 & \\
\hline & PS sludge & & & - & - & - & 9349 & \\
\hline & BT effluent & Oct 2019 & Sunny & 25.0 & 6.5 & 36.5 & 767 & \\
\hline & FS sludge & & & - & - & - & 3415 & \\
\hline & Effluent & & & 23.3 & 6.7 & 35 & 2.0 & \\
\hline & Dehydrated sludge & & & - & - & - & - & \\
\hline
\end{tabular}

STP: sewage treatment plant, PS: primary sedimentation, BT: biological treatment, FS: final sedimentation. 


\section{Clean-up and fractionation}

DLCs. An aliquot of crude extract was evaporated and transferred from toluene to dichloromethane. Dichloromethane fraction equivalent to $0.2 \mathrm{~L}$ of water or sludge sample or to $0.05 \mathrm{~g}$ of hydrated sludge sample was loaded onto the clean-up column and eluted with n-hexane. The eluate was then evaporated and loaded onto the fractionation column. The first fraction (designated 'chlorinated dioxin fraction'), which was eluted with $n$-hexane, contained chlorinated polychlorinated dibenzo-p-dioxins and dibenzofurans (PCDD/DFs) and dioxin-like polychlorinated biphenyls. The second fraction (designated 'brominated dioxin fraction'), which was eluted with $4 \%(\mathrm{v} / \mathrm{v})$ acetone/ $n$-hexane, contained PBDD/DFs. Finally, each fraction was evaporated under a gentle stream of nitrogen, and the residue was re-dissolved in dimethyl sulfoxide (DMSO) and used for the DR-CALUX assay.

PBDD/DFs. An aliquot of crude extract with high dioxin-like activity was spiked with a mixture of 13C12-labeled PBDD/DFs (ED-5408, Cambridge Isotope Laboratories, Inc.), and the solvent was changed from toluene to $n$-hexane. An n-hexane fraction equivalent to $1 \mathrm{~L}$ of water sample was cleaned-up by treatment with sulfuric acid, application to a $44 \%$ sulfuric acid $/ 10 \%$ silver nitrate-impregnated silica gel column, and elution with $20 \%$ (v/v) acetone/n-hexane. The eluate was then evaporated and loaded onto an activated carbon-impregnated silica gel column; the column was eluted with dichloromethane followed by toluene. The toluene fraction containing PBDD/DFs was evaporated, and the residue was re-dissolved in nonane containing a 13C12-labeled PBDD/DF syringe spike (ED-5409, Cambridge Isotope Laboratories, Inc., MA, U.S.) for subsequent GC-HRMS analysis.

FRs. An aliquot of crude extract was subjected to DMSO- $n$-hexane partitioning extraction. The DMSO fraction was mixed with sodium chloride solution and the mixture was extracted using ethyl acetate. The extract was then split into two portions and used for GC-APCI-QTOF-MS or LC-ESI-QTOF-MS analysis. For GC-APCIQTOF-MS analysis, the ethyl acetate extract was spiked with 13C12-labeled PBDEs, 13C6-hexabromobenzene, and 13C12-1,2-bis(2,4,6-tribromophenoxy) ethane (Wellington Laboratories, Inc., Ontario, Canada) and then loaded onto a column packed with silica gel. For LC-ESI-QTOF-MS analysis, ethyl acetate extract was spiked with 13C12-1,2,5,6,9,10-hexabromocyclododecane (HBCD), 13C12-tetrabromobisphenol A, D12-tris(2-chloroethyl) phosphate, D15-triphenyl phosphate (TPHP), D21tris(methylphenyl) phosphate, D27-tris-n-butyl phosphate, and D9-tris(dimethylphenyl) 
phosphate (TDMPP) (Wellington Laboratories, Inc.) and then loaded onto a column packed with octadecyl-bonded silica gel.

\section{GC-HRMS measurement}

PBDD/DFs. An HP-6890N gas chromatograph (Agilent, USA) equipped with a JMS700 mass spectrometer (JEOL, Japan) was used for the GC-HRMS measurement of PBDD/DFs. The electron-impact-selected-ion-monitoring method was used at a resolution of 10,000. For the measurements of TeBDD/DFs to HxBDD/DFs and HpBDD/DFs to OBDD/Fs, DB17-HT (30 $\mathrm{m} \times 0.25 \mathrm{~mm}$ i.d., $0.15 \mu \mathrm{m}$ film thickness, Agilent J\&W, USA) and DB-5MS (15 m $\times 0.25 \mathrm{~mm}$ i.d., $0.1 \mu \mathrm{m}$ film thickness, Agilent $\mathrm{J} \& \mathrm{~W})$ capillary columns were used, respectively. Six PBDD isomers $(2,3,7,8-\mathrm{TeBDD}$, 1,2,3,7,8-PeBDD, 1,2,3,4,7,8-HxBDD, 1,2,3,7,8,9-HxBDD, 1,2,3,4,6,7,8-HpBDD, and OBDD) and six PBDF isomers (2,3,7,8-TeBDF, 1,2,3,7,8-PeBDF, 2,3,4,7,8-PeBDF, 1,2,3,4,7,8-HxBDF, 1,2,3,4,6,7,8-HpBDF, and OBDF) were identified and quantified by the isotope dilution method using 13C12-labeled isomers.

\section{GC-APCI- and LC-ESI-QTOF-MS measurement}

FRs. An atmospheric-pressure chemical ionization interface for a gas chromatograph (GC-APCI interface, Agilent,) was used with a liquid chromatograph-electrospray ionization-quadrupole time-of-flight mass spectrometer (1290 Infinity LC/6530 QTOFMS, Agilent) system for the determination of a broad array of FRs. For the GC-APCIand LC-ESI-QTOF-MS measurements, an Rtx-5 (15 m × $0.53 \mathrm{~mm}$ i.d., $0.1 \mu \mathrm{m}$, Restek, USA) and a ZORBAX Eclipse Plus C18 RRHD (100 mm $\times 2.1 \mathrm{~mm}$ i.d., $1.8 \mu \mathrm{m}$, Agilent) were used, respectively. Twelve PBDE congeners (BDE-28, -47, -99, -100, 153, -154, -183, -196, -197, -206, -207, and 209) and 24 halogenated FR congeners (HxBBz, BTBPE, DBDPE, TBBPA-BDBPE, TTBP-TAZ, BEH-TEBP, EH-TBB, OBTMPI, PBB-Acr, PBEB, PBT, PeBBz, $\alpha$-DBE-DBCH, $\beta$-DBE-DBCH, $\alpha$-TBCO, $\beta$ TBCO, TBP-AE, TBP-BAE, TBP-DBPE, TBX, TBCT, DBHCTD, syn-DP, anti-DP) were identified and quantified using GC-APCI-QTOF-MS. Three HBCD isomers, five halogenated FR congeners (246-TrBPh, TBBPA, TBBPS, TBBPS-BDBPE, TDBPTAZTO), and 20 organophosphorus FR congeners (TCEP, TCIPP, TDCIPP, TDBPP, TTBNPP, BCMP-BCEP, DEG-BDCIPP, TPHP, MPDPP, BMPPP, TMPP, EHDPP, TDMPP, TIPPP, PBDPP, BPA-BDPP, PBDMPP, TIBP, TNBP, TBOEP) were identified and quantified by LC-ESI-QTOF-MS. 
Table S5 Target compounds for GC-HRMS measurement

\begin{tabular}{|c|c|}
\hline Target compounds & Abbreviation \\
\hline $2,3,7,8$-Tetrabromodibenzo- $p$-dioxin & $2,3,7,8-\mathrm{TeBDD}$ \\
\hline 1,3,6,8-Tetrabromodibenzo- $p$-dioxin & $1,3,6,8-\mathrm{TeBDD}$ \\
\hline 1,3,7,9-Tetrabromodibenzo- $p$-dioxin & $1,3,7,9-\mathrm{TeBDD}$ \\
\hline 1,2,3,4-Tetrabromodibenzo- $p$-dioxin & $1,2,3,4-\mathrm{TeBDD}$ \\
\hline $1,3,7,8$-Tetrabromodibenzo- $p$-dioxin & $1,3,7,8-\mathrm{TeBDD}$ \\
\hline 1,2,3,7,8-Pentabromodibenzo- $p$-dioxin & $1,2,3,7,8-\mathrm{PeBDD}$ \\
\hline 1,2,3,4,7,8-Hexabromodibenzo- $p$-dioxin & $1,2,3,4,7,8-\mathrm{HxBDD}$ \\
\hline 1,2,3,6,7,8-Hexabromodibenzo- $p$-dioxin & $1,2,3,6,7,8-\mathrm{HxBDD}$ \\
\hline 1,2,3,7,8,9-Hexabromodibenzo- $p$-dioxin & $1,2,3,7,8,9-\mathrm{HxBDD}$ \\
\hline $1,2,3,4,6,7,8$-Heptabromodibenzo- $p$-dioxin & $1,2,3,4,6,7,8-\mathrm{HpBDD}$ \\
\hline Octabromodibenzo- $p$-dioxin & OBDD \\
\hline 2,3,7,8-Tetrabromodibenzofuran & $2,3,7,8-\mathrm{TeBDF}$ \\
\hline 1,2,3,7,8-Pentabromodibenzofuran & $1,2,3,7,8-\mathrm{PeBDF}$ \\
\hline 2,3,4,7,8-Pentabromodibenzofuran & $2,3,4,7,8-\mathrm{PeBDF}$ \\
\hline $1,2,3,4,7,8$-Hexabromodibenzofuran & $1,2,3,4,7,8-\mathrm{HxBDF}$ \\
\hline 1,2,3,4,6,7,8-Heptabromodibenzofuran & $1,2,3,4,6,7,8-\mathrm{HpBDF}$ \\
\hline Octabromodibenzofuran & OBDF \\
\hline
\end{tabular}


Table S6 Target compounds for GC-APCI-QTOF-MS measurement

\begin{tabular}{|c|c|}
\hline Target compounds & Abbreviation \\
\hline 2,4,4'-Tribromodiphenyl ether & BDE-28 \\
\hline 2,2',4,4'-Tetrabromodiphenyl ether & BDE-47 \\
\hline 2,2',4,4',5-Pentabromodiphenyl ether & BDE-99 \\
\hline $2,2^{\prime}, 4,4^{\prime}, 6$-Pentabromodiphenyl ether & BDE-100 \\
\hline 2,2',4,4',5,5'-Hexabromodiphenyl ether & BDE-153 \\
\hline $2,2^{\prime}, 4,4^{\prime}, 5,6^{\prime}$-Hexabromodiphenyl ether & BDE-154 \\
\hline $2,2^{\prime}, 3,4,4^{\prime}, 5^{\prime}, 6$-Heptabromodiphenyl ether & BDE-183 \\
\hline $2,2^{\prime}, 3,3^{\prime}, 4,4^{\prime}, 5,6-$-Octabromodiphenyl ether & BDE-196 \\
\hline $2,2^{\prime}, 3,3^{\prime}, 4,4^{\prime}, 6,6^{\prime}$-Octabromodiphenyl ether & BDE-197 \\
\hline $2,2^{\prime}, 3,3^{\prime}, 4,4^{\prime}, 5,5^{\prime}, 6-$ Nonabromodiphenyl ether & BDE-206 \\
\hline $2,2^{\prime}, 3,3^{\prime}, 4,4^{\prime}, 5,6,6^{\prime}-$ Nonabromodiphenyl ether & BDE-207 \\
\hline Decabromodiphenyl ether & BDE-209 \\
\hline Hexabromobenzene & $\mathrm{HxBBz}$ \\
\hline 1,2-Bis(2,4,6-tribromophenoxy) ethane & ВТВРЕ \\
\hline Decabromodiphenyl ethane & DBDPE \\
\hline TBBPA bis(2,3-dbromopropyl) ether & TBBPA-BDBPE \\
\hline 2,4,6-tris(2,4,6-tribromophenoxy)-1,3,5-triazine & TTBP-TAZ \\
\hline Bis(2-ethyl-1-hexyl)tetrabromophthalate & BEH-TEBP \\
\hline 2-Ethylhexyl-2,3,4,5-tetrabromobenzoate & EH-TBB \\
\hline Octabromotrimethylphenylindane & OBTMPI \\
\hline Pentabromobenzyl acrylate & PBB-Acr \\
\hline Pentabromoethylbenzene & PBEB \\
\hline Pentabromotoluene & PBT \\
\hline 1,2,3,4,5-Pentabromobenzene & $\mathrm{PeBBz}$ \\
\hline$\alpha$-Tetrabromoethylcyclohexane & $\alpha$-DBE-DBCH \\
\hline$\beta$-Tetrabromoethylcyclohexane & $\beta$-DBE-DBCH \\
\hline$\alpha-1,2,5,6$-Tetrabromocyclooctane & $\alpha-\mathrm{TBCO}$ \\
\hline$\beta-1,2,5,6$-Tetrabromocyclooctane & $\beta-\mathrm{TBCO}$ \\
\hline Allyl 2,4,6-tribromophenyl ether & TBP-AE \\
\hline 2-Bromoallyl-2,4,6-tribromophenyl ether & TBP-BAE \\
\hline 2,3-Dibromopropyl-2,4,6-tribromophenyl ether & TBP-DBPE \\
\hline 2,3,5,6-Tetrabromo- $p$-xylene & TBX \\
\hline 3,4,5,6-Tetrabromo-2-chlorotoluene & TBCT \\
\hline Hexachlorocyclopentenyl-dibromocyclooctane & DBHCTD \\
\hline syn-Dechlorane Plus & syn-DP \\
\hline anti-Dechlorane Plus & anti-DP \\
\hline
\end{tabular}


Table S7 Target compounds for LC-ESI-QTOF-MS measurement

\begin{tabular}{|c|c|}
\hline Target compound & Abbreviation \\
\hline$\alpha-1,2,5,6,9,10$-Hexabromocyclododecane & $\alpha-\mathrm{HBCD}$ \\
\hline$\beta-1,2,5,6,9,10$-Hexabromocyclododecane & $\beta$-HBCD \\
\hline$\gamma-1,2,5,6,9,10-$ Hexabromocyclododecane & $\gamma$-HBCD \\
\hline 2,4,6-Tribromophenol & 246-TrBPh \\
\hline Tetrabromobisphenol A & TBBPA \\
\hline Tetrabromobisphenol S & TBBPS \\
\hline TBBPS bis(2,3-dbromopropyl) ether & TBBPS-BDBPE \\
\hline Tris(2,3-dibromopropyl) isocyanurate & TDBP-TAZTO \\
\hline Tris(2-chloroethyl) phosphate & TCEP \\
\hline Tris(2-chloroisopropyl) phosphate & TCIPP \\
\hline Tris(1,3-dichloroisopropyl) phosphate & TDCIPP \\
\hline Tris(2,3-dibromopropyl) phosphate & TDBPP \\
\hline Tris(tribromoneopentyl) phosphate & TTBNPP \\
\hline 2,2-Bis(chloromethyl)-1,3-propanediol bis[di(2-chloroethyl) phosphate] & BCMP-BCEP \\
\hline Diethylene glycol bis[di(2-chloroisopropyl) phosphate] & DEG-BDCIPP \\
\hline Triphenyl phosphate & ТPHP \\
\hline Methylphenyl diphenyl phosphate & MPDPP \\
\hline Bis(methylphenyl) phenyl phosphate & ВМРРP \\
\hline 2-Ethylhexyldiphenyl phosphate & EHDPP \\
\hline Tris(methylphenyl) phosphate & TMPP \\
\hline Tris(dimethylphenyl) phosphate & TDMPP \\
\hline Tris(isopropylphenyl) phosphate & ТIPРP \\
\hline 1,3-Phenylene bis(diphenyl phosphate) & PBDPP \\
\hline Bisphenol A bis(diphenyl phosphate) & BPA-BDPP \\
\hline 1,3-Phenylene bis(dimethylphenyl phosphate) & PBDMPP \\
\hline Tris(isobutyl) phosphate & TIBP \\
\hline Tri-n-butyl phosphate & TNBP \\
\hline Tris(2-butoxyethyl) phosphate & TBOEP \\
\hline
\end{tabular}




\section{Quality assurance and quality control}

Dioxin-like activity. The luciferase inductions of $0.3,1.0,3.0$, and $10 \mathrm{pM} 2,3,7,8$ TCDD were $1.7 \pm 0.5 \%, 6.0 \pm 1.6 \%, 20.4 \pm 2.8 \%$, and $47.9 \pm 5.1 \%$ (average \pm S.D., $\mathrm{n}=$ 48). The induction value (luciferase activity of 300 pM 2,3,7,8-TCDD divided by the luciferase activity of DMSO) was $17.0 \pm 4.1$ (average \pm S.D., $n=48$ ). Based on these conditions, the limit of quantification (LOQ) was defined as 10 times the standard deviation of the DMSO vehicle-only control and approximately $1.0 \mathrm{pg} / \mathrm{L}$ for TCDDEQPBDD/DFs and TCDD-EQPCDD/DFs values in water and sludge samples or $0.010 \mathrm{ng} / \mathrm{kg}$ for TCDD-EQPBDD/DFs and TCDD-EQPCDD/DFs values in dehydrated sludge samples. Procedural blanks were measured with approximately $10 \%$ of tested samples. Whereas TCDD-EQPCDD/DFs values were not detected above the LOQ, TCDD-EQPBDD/DFs values equivalent to the LOQ were detected. These values were subtracted from the TCDDEQPBDD/DFs. Two of the tested samples were randomly selected and duplicated to evaluate the precision of the detection method. The relative percent differences were $20 \%$ to $24 \%$ and $2.0 \%$ to $12 \%$ for the TCDD-EQPBDD/DFs and TCDD-EQPCDD/DFs values, respectively.

PBDD/DFs. The LOQ was defined as a signal-to-noise ratio of 10 or $2.7-380 \mathrm{pg} / \mathrm{L}$ for $\mathrm{TeBDD} / \mathrm{DFs}$ to OBDD/DF. The concentrations of $\mathrm{PBDD} / \mathrm{DFs}$ in the procedural blanks were lower than the LOQ. Recoveries of the ${ }^{13} \mathrm{C}$-labeled PBDD/DFs in spiked extracts $(\mathrm{n}=7)$ ranged from $50.8 \%$ to $105.4 \%$.

FRs. The LOQ was defined as a signal-to-noise ratio of 10 or $2-200 \mathrm{ng} / \mathrm{L}$ for the FRs. The concentrations of the FRs in the procedural blanks were lower than the LOQ. 
Table S8 Dioxin-like compounds in the filtrate and suspended solid fractions of effluent samples collected from the deca-BDE handling facilities

\begin{tabular}{|c|c|c|c|c|c|c|c|c|c|c|c|c|c|c|c|}
\hline \multirow{2}{*}{ Compound } & \multirow{2}{*}{$\begin{array}{l}\text { DHF-1 } \\
\text { EF-1 }\end{array}$} & \multirow{2}{*}{$\begin{array}{l}\text { DHF-2 } \\
\text { EF-1 }\end{array}$} & \multirow{2}{*}{$\begin{array}{l}\text { DHF-3 } \\
\text { EF-1 }\end{array}$} & \multirow{2}{*}{$\begin{array}{l}\text { DHF-4 } \\
\text { EF-1 }\end{array}$} & \multirow{2}{*}{$\begin{array}{l}\text { DHF-5 } \\
\text { EF-1 }\end{array}$} & \multirow{2}{*}{$\begin{array}{l}\text { DHF-6 } \\
\text { EF-1 }\end{array}$} & \multirow{2}{*}{$\begin{array}{l}\text { DHF-7 } \\
\text { EF-1 }\end{array}$} & \multicolumn{2}{|c|}{ DHF-8 } & \multicolumn{2}{|c|}{ DHF-9 } & \multicolumn{2}{|c|}{ DHF-10 } & \multicolumn{2}{|c|}{ DHF-11 DHF-12 } \\
\hline & & & & & & & & EF-1 & EF-2 & EF-1 & $\mathrm{EF}-2$ & EF-1 & EF-2 & $\mathrm{EF}-1$ & EF-1 \\
\hline \multicolumn{16}{|l|}{ Concentration $(\mathrm{pg} / \mathrm{L})$} \\
\hline \multicolumn{16}{|l|}{ TCDD-EQ } \\
\hline Filtrate $(<0.5 \mu \mathrm{m})$ & 1.6 & 1.3 & 2.7 & 27 & 3.6 & 21 & $<1.0$ & 3.7 & 28 & 320 & 110 & 18 & 44 & $<1.0$ & 1.3 \\
\hline Suspended solid $(>0.5 \mu \mathrm{m})$ & 1.5 & $<1.0$ & 160 & 26 & 19 & 2.0 & 7.2 & 670 & 10 & 540 & 92 & 37 & 22 & 3.7 & 1.7 \\
\hline Filtrate $(<0.5 \mu \mathrm{m})$ & $<1.0$ & $<1.0$ & 1.3 & 3.7 & $<1.0$ & 5.8 & $<1.0$ & 2.0 & 5.7 & 13 & $<1.0$ & 3.2 & 3.1 & $<1.0$ & $<1.0$ \\
\hline Suspended solid $(>0.5 \mu \mathrm{m})$ & $<1.0$ & $<1.0$ & 4.4 & 2.1 & $<1.0$ & 1.1 & $<1.0$ & 76 & 2.4 & 14 & $<10$ & 13 & 2.8 & 1.4 & 1.1 \\
\hline \multicolumn{16}{|l|}{ Contribution $(\%)$} \\
\hline \multicolumn{16}{|l|}{ TCDD-EQ } \\
\hline Filtrate $(<0.5 \mu \mathrm{m})$ & 52 & 100 & 2 & 51 & 16 & 91 & 0 & 1 & 74 & 37 & 54 & 33 & 67 & 0 & 43 \\
\hline Suspended solid $(>0.5 \mu \mathrm{m})$ & 48 & 0 & 98 & 49 & 84 & 9 & 100 & 99 & 26 & 63 & 46 & 67 & 33 & 100 & 57 \\
\hline \multicolumn{16}{|l|}{ TCDD-EQ ${ }_{\mathrm{PCDD} / \mathrm{DFs}}$} \\
\hline Filtrate $(<0.5 \mu \mathrm{m})$ & NA & NA & 23 & 64 & NA & 84 & NA & 3 & 70 & 48 & NA & 20 & 53 & 0 & 0 \\
\hline Suspended solid $(>0.5 \mu \mathrm{m})$ & NA & NA & 77 & 36 & NA & 16 & NA & 97 & 30 & 52 & NA & 80 & 47 & 100 & 100 \\
\hline
\end{tabular}

NA: not available, DHF: deca-BDE handling facility, EF-1: effluent collected during December 2017 and February 2018, EF-2: effluent collected in October 2019, TCDD-EQPBDD/DFs: TCDD equivalent for the brominated dioxin fraction, TCDD-EQPCDD/DFs: TCDD equivalent for the chlorinated dioxin fraction. 
Table S9 PBDD/DFs (pg/L) in effluent samples ${ }^{\mathrm{a}}$ with a high dioxin-like activity collected from deca-BDE handling facilities

\begin{tabular}{|c|c|c|c|c|c|c|c|}
\hline Compound & WHO-TEF ${ }^{\mathrm{b}}$ & CALUX-REP ${ }^{\mathrm{c}}$ & $\begin{array}{c}\text { DHF-3 } \\
\text { EF-1 }\end{array}$ & $\begin{array}{c}\text { DHF-4 } \\
\text { EF-1 }\end{array}$ & $\begin{array}{c}\text { DHF-8 } \\
\text { EF-1 }\end{array}$ & $\begin{array}{c}\text { DHF-9 } \\
\text { EF-1 }\end{array}$ & $\begin{array}{c}\text { DHF-10 } \\
\text { EF-1 }\end{array}$ \\
\hline \multicolumn{8}{|l|}{ Isomers } \\
\hline \multicolumn{8}{|l|}{ PBDDs } \\
\hline $2,3,7,8-\mathrm{TeBDD}$ & 1 & 0.3 & $<13$ & $<2.7$ & $<9$ & $<13$ & $<2.7$ \\
\hline 1,3,6,8-TeBDD & & & 20 & $<2.7$ & 9 & 15 & $<2.7$ \\
\hline 1,3,7,9-TeBDD & & & $<13$ & $<2.7$ & $<9$ & 15 & $<2.7$ \\
\hline 1,2,3,4-TeBDD & & & $<13$ & $<2.7$ & $<9$ & $<13$ & $<2.7$ \\
\hline 1,3,7,8-TeBDD & & & $<13$ & $<2.7$ & $<9$ & $<13$ & $<2.7$ \\
\hline $1,2,3,7,8-\mathrm{PeBDF}$ & 1 & 0.083 & $<39$ & $<8$ & $<26$ & $<39$ & $<8$ \\
\hline $1,2,3,4,7,8-\mathrm{HxBDD}$ & 0.1 & 0.023 & $<200$ & $<40$ & $<130$ & $<200$ & $<40$ \\
\hline $1,2,3,6,7,8-\mathrm{HxBDD}$ & 0.1 & 0.0024 & $<240$ & $<48$ & $<160$ & $<240$ & $<48$ \\
\hline $1,2,3,7,8,9-\mathrm{HxBDD}$ & 0.1 & 0.0080 & $<180$ & $<37$ & $<120$ & $<180$ & $<37$ \\
\hline $1,2,3,4,6,7,8-\mathrm{HpBDD}$ & 0.01 & 0.0037 & $<150$ & $<30$ & $<100$ & $<150$ & $<30$ \\
\hline OBDD & 0.003 & 0.00013 & 850 & $<77$ & $<250$ & $<380$ & $<77$ \\
\hline \multicolumn{8}{|l|}{ PBDFs } \\
\hline $2,3,7,8-\mathrm{TeBDF}$ & 0.1 & 0.51 & $<14$ & $<2.8$ & 50 & $<14$ & 3.7 \\
\hline $1,2,3,7,8-\mathrm{PeBDF}$ & 0.03 & 0.095 & $<62$ & $<13$ & $<41$ & $<62$ & $<13$ \\
\hline $2,3,4,7,8-\mathrm{PeBDF}$ & 0.3 & 0.20 & $<79$ & $<16$ & $<53$ & $<71$ & $<16$ \\
\hline $1,2,3,4,7,8-\mathrm{HxBDF}$ & 0.1 & 0.010 & $<170$ & $<34$ & $<110$ & $<170$ & $<34$ \\
\hline $1,2,3,4,6,7,8-\mathrm{HpBDF}$ & 0.01 & 0.0014 & 44,000 & 31 & 330 & 10,000 & 420 \\
\hline OBDF & 0.0003 & 0.00012 & 360,000 & $<86$ & $<280$ & 92,000 & 1,800 \\
\hline \multicolumn{8}{|l|}{ Homologue } \\
\hline TeBDDs & & & 20 & $<2.7$ & 20 & 29 & $<2.7$ \\
\hline PeBDDs & & & $<39$ & $<8$ & 10 & $<39$ & $<8.0$ \\
\hline HxBDDs & & & $<200$ & $<41$ & $<140$ & $<200$ & $<42$ \\
\hline HpBDDs & & & $<150$ & $<30$ & $<100$ & $<150$ & $<30$ \\
\hline OBDD & & & 850 & $<77$ & $<250$ & $<380$ & $<77$ \\
\hline TeBDFs & & & 86 & $<2.8$ & 150 & $<14$ & 100 \\
\hline PeBDFs & & & 290 & $<14$ & 92 & $<71$ & 130 \\
\hline HxBDFs & & & $<170$ & $<34$ & 270 & $<170$ & 180 \\
\hline HpBDFs & & & 44,000 & 31 & 330 & 10,000 & 420 \\
\hline OBDF & & & 360,000 & $<86$ & $<280$ & 92,000 & 1,800 \\
\hline Total PBDD/DFs & & & 410,000 & 31 & 880 & 100,000 & 2,630 \\
\hline WHO-TEQ ${ }_{\text {PBDDs }}{ }^{\mathrm{d}}$ & & & 2.6 & 0.25 & 0 & 0 & 0.14 \\
\hline WHO-TEQ ${ }_{\mathrm{PBDFs}}{ }^{\mathrm{d}}$ & & & 548 & 0.23 & 8.3 & 130 & 6.9 \\
\hline WHO-TEQ $_{\text {PBDD/DFs }}{ }^{\mathrm{d}}$ & & & 550 & 0.48 & 8.3 & 130 & 7.0 \\
\hline Theoretical TCDD-EQ ${ }_{\text {PBDDs }}{ }^{\mathrm{e}}$ & & & 0.11 & 0.091 & 0 & 0 & 0.051 \\
\hline Theoretical TCDD-EQ ${ }_{\mathrm{PBDDF}}{ }^{\mathrm{e}}$ & & & 100 & 0.031 & 26 & 24 & 2.8 \\
\hline Theoretical TCDD-EQPBDD/DFs ${ }^{\mathrm{e}}$ & & & 100 & 0.12 & 26 & 24 & 2.9 \\
\hline
\end{tabular}

${ }^{a}$ Combined fraction (filtrate + suspended solid), ${ }^{\mathrm{b}} \mathrm{WHO}$-toxicity equivalency factor (TEF) was cited from van den Berg et al. (2006), ${ }^{\mathrm{c}}$ CALUX-REP was cited from Suzuki et al. ${ }^{7}$. Average value between REP-EC 5 5TCD (mass basis) and REP-EC 20 (mass basis) was used in this study. ${ }^{\mathrm{d}} \mathrm{PBDD} / \mathrm{DF}$ sere calculated by multiplying the concentration of each individual PBDD/DFs by the WHO-TEFs of their chlorinated analogues, and then adding the individual WHO-TEQs to obtain the total WHO-TEQ. ${ }^{\text {e }}$ PBDD/DFs were calculated by multiplying the concentration of each individual PBDD/DF by the respective CALUX-REP of its chlorinated analogue, and then adding the individual theoretical TCDD-EQ to obtain theoretical TCDD-EQ.

DHF: deca-BDE handling facility, EF-1: effluent collected in December 2017 or February 2018. 


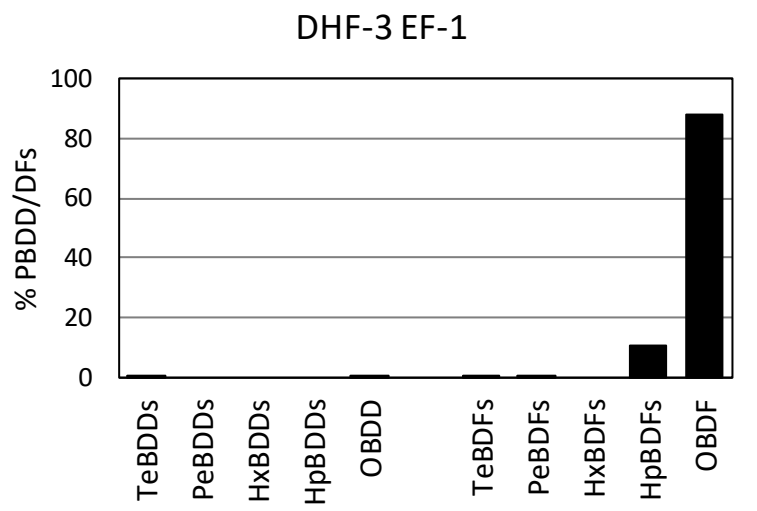

DHF-9 EF-1

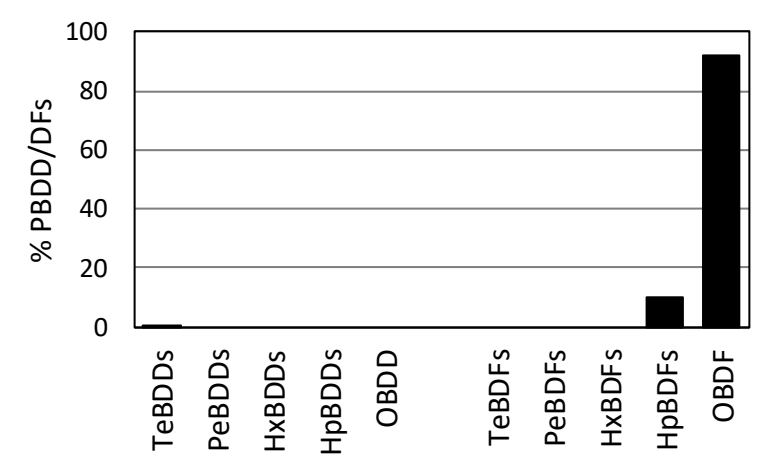

DHF-4 EF-1

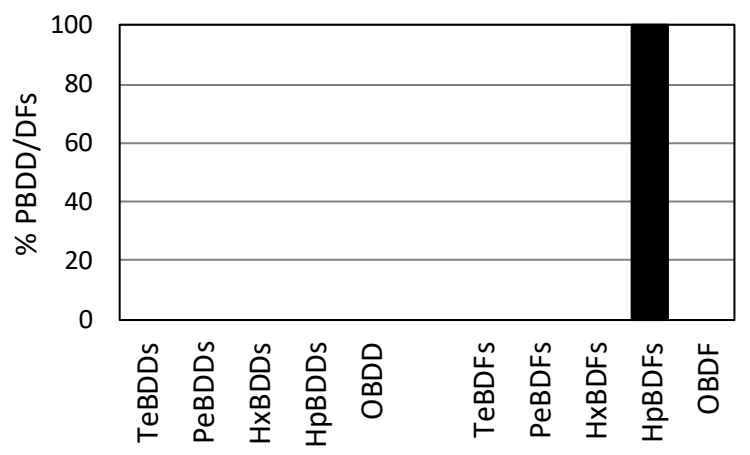

DHF-10 EF-1

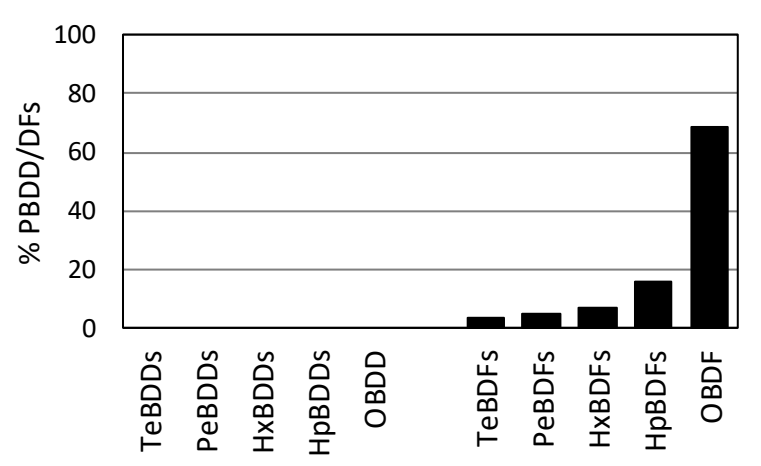

DHF-8 EF-1

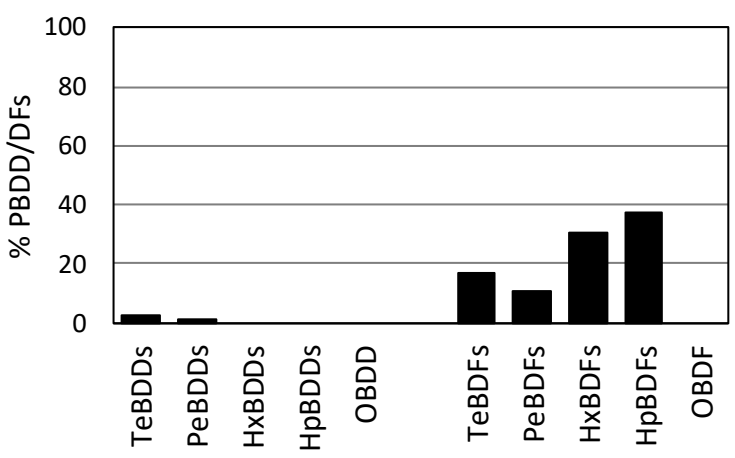

Figure S2 Homolog profile of PBDD/DFs in effluents from deca-BDE handling facilities 3, 4, 8, 9, and 10, which showed a high dioxin-like compound activity

DHF: deca-BDE handling facility, EF-1: effluent collected in December 2017 and February 2018. 
Table S10 Dioxin-like compounds in the filtrate and suspended solids fractions of effluent and sludge samples from sewage treatment plants receiving effluent from a deca-BDE handling facility

\begin{tabular}{|c|c|c|c|c|c|c|c|c|c|c|}
\hline \multirow{2}{*}{ Compound } & \multicolumn{2}{|c|}{ STP-1 } & \multicolumn{2}{|c|}{ STP-2 } & \multicolumn{6}{|c|}{ STP-3 } \\
\hline & INF & $\mathrm{EF}$ & INF & EF & INF & PS EF & BT EF & EF & PS sludge & FS sludge \\
\hline \multicolumn{11}{|l|}{ Concentration (pg/L) } \\
\hline \multicolumn{11}{|l|}{ TCDD-EQ } \\
\hline Filtrate $(<0.5 \mu \mathrm{m})$ & 7.6 & $<1.0$ & $<1.0$ & $<1.0$ & 2.0 & 26 & $<1.0$ & $<1.0$ & $<1.0$ & $<1.0$ \\
\hline Suspended solid $(>0.5 \mu \mathrm{m})$ & 13 & $<1.0$ & 5.5 & 1.8 & 83 & 26 & 180 & 2.0 & 10,000 & 860 \\
\hline \multicolumn{11}{|l|}{ TCDD-EQ ${ }_{\mathrm{PCDD} / \mathrm{DFs}}$} \\
\hline Filtrate $(<0.5 \mu \mathrm{m})$ & 5.4 & $<1.0$ & 1.2 & $<1.0$ & $<1.0$ & $<1.0$ & $<1.0$ & $<1.0$ & $<1.0$ & $<1.0$ \\
\hline Suspended solid $(>0.5 \mu \mathrm{m})$ & 2.6 & $<1.0$ & $<1.0$ & $<1.0$ & 3.1 & 2.2 & 41 & 1.0 & 450 & 73 \\
\hline \multicolumn{11}{|l|}{ Contribution (\%) } \\
\hline \multicolumn{11}{|l|}{ TCDD-EQ } \\
\hline Filtrate $(<0.5 \mu \mathrm{m})$ & 37 & NA & 0 & 0 & 2 & 50 & 0 & 0 & 0 & 0 \\
\hline Suspended solid $(>0.5 \mu \mathrm{m})$ & 63 & NA & 100 & 100 & 98 & 50 & 100 & 100 & 100 & 100 \\
\hline \multicolumn{11}{|l|}{ TCDD-EQ ${ }_{\mathrm{PCDD} / \mathrm{DFs}}$} \\
\hline Filtrate $(<0.5 \mu \mathrm{m})$ & 68 & NA & 100 & NA & 0 & 0 & 0 & 0 & 0 & 0 \\
\hline Suspended solid $(>0.5 \mu \mathrm{m})$ & 33 & NA & 0 & NA & 100 & 100 & 100 & 100 & 100 & 100 \\
\hline
\end{tabular}

NA: not available, STP: sewage treatment plant, INF: influent, EF: effluent, PS: primary sedimentation, BT: biological treatment, FS: final sedimentation, D: dehydrated, TCDD-EQPBDD/DFs: TCDD equivalent for the brominated dioxin fraction, TCDD-EQPCDD/DFs: TCDD equivalent for the chlorinated dioxin fraction. 
Table S11 PBDD/DFs in water samples ${ }^{\mathrm{a}}$ and sludge collected from Japan and USA

\begin{tabular}{|c|c|c|c|c|c|c|}
\hline Sample & Location & Country & Year & $\mathrm{n}$ & Concentration & Reference \\
\hline \multicolumn{7}{|c|}{ Water sample (pg WHO-TEQ/L) } \\
\hline Runoff water & Ground Zero & USA & 2001 & 2 & $110-960$ & Litten et al., 2003 \\
\hline Effluent & Deca-BDE handling facility (Textile) & Japan & 2003 & 3 & $3.6-130$ & MOEJ, 2003 \\
\hline Effluent & Deca-BDE handling facility (Plastic manufacturing) & Japan & 2004 & 6 & ND-14 & MOEJ, 2004 \\
\hline Effluent & Sewage treatment plant & Japan & 2004 & 3 & ND-2.2 & MOEJ, 2004 \\
\hline Effluent & Deca-BDE handling facility (Textile) & Japan & 2006 & 4 & $0.37-19$ & MOEJ, 2006 \\
\hline Effluent & Deca-BDE handling facility (Textile) & Japan & 2013 & 5 & $2-2500$ & MOEJ, 2013 \\
\hline Effluent & Sewage treatment plant & Japan & 2014 & 6 & $0.0043-3.2$ & MOEJ, 2014 \\
\hline Effluent & Deca-BDE handling facility (Textile) & Japan & 2015 & 2 & $0.59-36$ & MOEJ, 2015 \\
\hline \multicolumn{7}{|c|}{ Sludge (ng WHO-TEQ/g d.w.) } \\
\hline Biosolid & Wastewater treatment plant & USA & 2001 & 5 & $0.015-0.672$ & Venkatesan and Halden, 2014 \\
\hline Sewage sludge & Sewage treatment plant & Japan & 2004 & 3 & ND-2.2 & MOEJ, 2004 \\
\hline Sewage sludge & Sewage treatment plant & Japan & 2014 & 2 & $0.0013-7.4$ & MOEJ, 2014 \\
\hline
\end{tabular}

${ }^{\mathrm{a}}$ Combined fraction (filtrate + suspended solid).

ND: not detected. 
Table S12 PBDEs in water samples ${ }^{\mathrm{a}}$ and sludge collected from various countries

\begin{tabular}{|c|c|c|c|c|c|c|c|c|}
\hline Sample & Location & Country & Year & $\mathrm{n}$ & Compound & Concentration & Main isomer & Reference \\
\hline \multicolumn{9}{|l|}{ Water sample (ng/L) } \\
\hline Surface water & Coastal ocean & Hong Kong & 2005 & 10 & Tri-deca-BDE & ND-297.3 & BDE-28, 47, 100, 183 & Wurl et al., 2006 \\
\hline Surface water & River delta and coastal ocean & China & 2005-2006 & 96 & Tri-deca-BDE & $0.344-68$ & BDE-209 & Guan et al., 2007 \\
\hline Surface water & Arctic ocean & - & 2005-2008 & 140 & Tri-deca-BDE & $0.0009-0.0112$ & BDE-209 & Salvadó et al., 2016 \\
\hline Surface water & Lake & Korea & 2008 & 29 & Tri-deca-BDE & $0.16-3.2$ & BDE-209 & Moon et al., 2012 \\
\hline Surface water & The European Arctic & - & 2009 & 16 & Tri-deca-BDE & $0.000005-0.00064$ & BDE-47, 99 & Möller et al., 2011a \\
\hline Surface water & The East Asia to the Arctic & - & 2010 & 14 & Tetra-deca-BDE & ND-0.000809 & BDE-47, 99 & Möller et al., 2011b \\
\hline Surface water & Harbor and estuary & Japan & 2010 & 49 & Tetra-deca-BDE & $<0.11-14$ & BDE-209 & MOEJ, 2011 \\
\hline Surface water & River at upstream of MSTP & China & 2011 & 6 & Tri-deca-BDE & $8.474 \pm 2.750$ & BDE-209 & Wang et al., 2013 \\
\hline Surface water & River at downstream of MSTP & China & 2011 & 6 & Tri-deca-BDE & $32.876 \pm 3.464$ & BDE-209 & Wang et al., 2013 \\
\hline Surface water & Harbor and estuary & Japan & 2011 & 49 & Tetra-deca-BDE & $<0.031-59$ & BDE-209 & MOEJ, 2012 \\
\hline Surface water & River & UK & 2011 & 13 & Deca-BDE & $17-295$ & BDE-209 & Cristale et al., 2013 \\
\hline Surface water & Harbor and estuary & Japan & 2012 & 48 & Tetra-deca-BDE & $<0.24-12$ & BDE-209 & MOEJ, 2013 \\
\hline Surface water & Harbor and estuary & Japan & 2014 & 48 & Tetra-deca-BDE & $<0.021-6.2$ & BDE-209 & MOEJ, 2015 \\
\hline Surface water & Harbor and estuary & Japan & 2015 & 48 & Tetra-deca-BDE & $0.16-13$ & BDE-209 & MOEJ, 2016 \\
\hline Surface water & Harbor and estuary & Japan & 2016 & 48 & Tetra-deca-BDE & $<0.014-38$ & BDE-209 & MOEJ, 2017 \\
\hline Surface water & Harbor and estuary & Japan & 2017 & 47 & Tetra-deca-BDE & $<0.024-4.6$ & BDE-209 & MOEJ, 2018 \\
\hline Surface water & Harbor and estuary & Japan & 2018 & 47 & Tetra-deca-BDE & $<0.019-3.2$ & BDE-209 & MOEJ, 2019 \\
\hline Laundry wastewater & Family home & USA & 2011-2012 & 20 & Tri-deca-BDE & $332-68,100$ & BDE-47, 99 & Schreder and La Guardia, 2014 \\
\hline Raw water & Wastewater treatment plant & Australia & 2007 & 3 & Tri-deca-BDE & $42-100$ & BDE-47, 99, 209 & Clarke et al., 2010 \\
\hline Raw water & Sewage treatment plant & China & 2006-2007 & 5 & Tri-deca-BDE & $13.3-2496.4$ & BDE-209 & Peng et al., 2009 \\
\hline Raw water & Wastewater treatment plant & South Africa & $2010-2011$ & 6 & Tri-deca-BDE & $48.8-401$ & BDE-47, 209 & Daso et al., 2012 \\
\hline Influent & Wastewater treatment plant & China & 2009-2016 & 32 & Deca-BDE & $9.2-1050$ & BDE-209 & Li et al., 2018 \\
\hline Influent & Wastewater treatment plant & China & 2010-2011 & 3 & Tri-deca-BDE & $3.25-5.35$ & BDE-209 & Xiang et al., 2013 \\
\hline Influent & Municipal sewage treatment plant & China & 2011 & 6 & Tri-deca-BDE & $36.884 \pm 19.530$ & BDE-209 & Wang et al., 2013 \\
\hline Influent & Wastewater treatment plant & USA & 2011-2012 & 2 & Tri-deca-BDE & $169-243$ & BDE-209 & Schreder and La Guardia, 2014 \\
\hline Influent & Wastewater treatment plant & Hong Kong & 2011-2013 & 60 & Tri-deca-BDE & $1-254$ & BDE-47, 209 & Deng et al., 2015 \\
\hline Effluent & Sewage treatment plant & China & 2006-2007 & 5 & Tri-deca-BDE & $0.9-4.4$ & BDE-209 & Peng et al., 2009 \\
\hline Effluent & Wastewater treatment plant & Australia & 2007 & 3 & Tri-deca-BDE & $0.14-0.71$ & BDE-47, 99, 209 & Clarke et al., 2010 \\
\hline Effluent & Municipal sewage treatment plant & China & 2011 & 6 & Tri-deca-BDE & $188.578 \pm 81.255$ & BDE-209 & Wang et al., 2013 \\
\hline Effluent & Wastewater treatment plant & China & 2009-2016 & 32 & Deca-BDE & $9.5-68.6$ & BDE-209 & Li et al., 2018 \\
\hline Effluent & Wastewater treatment plant & China & 2010-2011 & 3 & Tri-deca-BDE & $2.35-3.89$ & BDE-209 & Xiang et al., 2013 \\
\hline Effluent & Wastewater treatment plant & South Africa & 2010-2011 & 6 & Tri-deca-BDE & $16.4-1240$ & BDE-47, 209 & Daso et al., 2012 \\
\hline Effluent & Wastewater treatment plant & USA & 2011-2012 & 2 & Tri-deca-BDE & $16.3-40$ & BDE-209 & Schreder and La Guardia, 2014 \\
\hline Effluent & Wastewater treatment plant & Hong Kong & 2011-2013 & 60 & Tri-deca-BDE & $2-230$ & BDE-47, 99 & Deng et al., 2015 \\
\hline \multicolumn{9}{|l|}{ Sludge (ng/g d.w.) } \\
\hline Biosolid & Wastewater treatment plant & Australia & 2007 & 3 & Tri-deca-BDE & $270-340$ & BDE-47, 99, 209 & Clarke et al., 2010 \\
\hline Dewatered sludge & Sewage treatment plant & China & 2006-2007 & 3 & Tri-deca-BDE & $158-23,750$ & BDE-209 & Peng et al., 2009 \\
\hline Dewatered sludge & Wastewater treatment plant & China & 2009-2016 & 24 & Deca-BDE & $142.8-607.0$ & BDE-209 & Li et al., 2018 \\
\hline Dewatered sludge & Wastewater treatment plant & South Africa & $2010-2011$ & 6 & Tri-deca-BDE & $50.2-1160$ & BDE-47, 209 & Daso et al., 2012 \\
\hline Dewatered sludge & Wastewater treatment plant & Hong Kong & 2011-2013 & 60 & Tri-deca-BDE & $9-307$ & BDE-47, 209 & Deng et al., 2015 \\
\hline Sewage sludge & Wastewater treatment plant & China & 2005 & 31 & Tri-deca-BDE & $5.1-1114.9$ & BDE-209 & Wang et al., 2007 \\
\hline
\end{tabular}

${ }^{a}$ Combined fraction (Filtrate + suspended solid).

ND: not detected. 
Table S13 Other FRs in water samples ${ }^{\mathrm{a}}$ and sludge collected from various countries

\begin{tabular}{|c|c|c|c|c|c|c|c|}
\hline Sample & Location & Country & Year & $\mathrm{n}$ & Compound & Concentration & Reference \\
\hline \multicolumn{8}{|l|}{ Water sample (ng/L) } \\
\hline Surface water & River & UK & 2011 & 13 & DBDPE & $113-26,050$ & Cristale et al., 2013 \\
\hline Effluent & Wastewater treatment plant & China & $2009-2016$ & 32 & DBDPE & ND-21.8 & Li et al., 2018 \\
\hline Laundry wastewater & Family home & USA & $2011-2012$ & 20 & DBDPE & ND-193 & Schreder and La Guardia, 2014 \\
\hline Influent & Wastewater treatment plant & China & 2009-2016 & 32 & DBDPE & $0.2-86.1$ & Li et al., 2018 \\
\hline Influent & Wastewater treatment plant & Canada & - & 145 & DBDPE & ND-130 & Kim et al., 2014 \\
\hline Surface water & River & Germany & 2002 & & TCIPP & $20-200$ & Andresen et al., 2004 \\
\hline Laundry wastewater & Family home & USA & $2011-2012$ & 20 & TCIPP & $3,530-561,100$ & Schreder and La Guardia, 2014 \\
\hline Influent & Wastewater treatment plant & USA & $2011-2012$ & 2 & TCIPP & $1,380-2,090$ & Schreder and La Guardia, 2014 \\
\hline Influent & Wastewater treatment plant & Spain & 2012 & 5 & TCIPP & $1,700-6,750$ & Cristale et al., 2016 \\
\hline Effluent & Wastewater treatment plant & USA & $2011-2012$ & 2 & TCIPP & $7,010-8,380$ & Schreder and La Guardia, 2014 \\
\hline Effluent & Wastewater treatment plant & Spain & 2012 & 5 & TCIPP & $2400-3700$ & Cristale et al., 2016 \\
\hline Surface water & Lake & USA & 2013-2014 & 292 & TBOEP & ND-38,700 & Elliott et al., 2017 \\
\hline Influent & Wastewater treatment plant & Spain & 2012 & 5 & TBOEP & $1,560-46,000$ & Cristale et al., 2016 \\
\hline Effluent & Wastewater treatment plant & Spain & 2012 & 5 & TBOEP & $207-3600$ & Cristale et al., 2016 \\
\hline \multicolumn{8}{|l|}{ Sludge (ng/g d.w.) } \\
\hline Dewatered sludge & Wastewater treatment plant & Canada & - & 104 & DBDPE & ND-220 & Kim et al., 2014 \\
\hline Dewatered sludge & Wastewater treatment plant & China & 2009-2016 & 24 & DBDPE & $75.1-607.3$ & Li et al., 2018 \\
\hline Sludge & Wastewater treatment plant & Spain & 2012 & 5 & TBOEP & ND & Cristale et al., 2016 \\
\hline Sludge & Sewage treatment plant & China & 2014 & 6 & BPA-BDPP & $2.06-5.82$ & Liang et al., 2018 \\
\hline Sludge & Sewage treatment plant & China & 2014 & 6 & PBDPP & $0.44-3.45$ & Liang et al., 2018 \\
\hline
\end{tabular}

${ }^{a}$ Combined fraction (Filtrate + suspended solid).

ND: not detected. 
Table S14 Short- and long-term toxicity of 2,3,7,8-TCDD and FRs in fish, crustaceans, and algae

\begin{tabular}{|c|c|c|c|c|c|c|}
\hline Compound & & Species & Exposure duration & Criterion & Value $(\mathrm{mg} / \mathrm{L})$ & Reference \\
\hline 2,3,7,8-TCDD & Fish & Medaka (Oryzias latipes) & $6 \mathrm{~h}^{\mathrm{a}}$ & NOEC & 0.00000329 & Nakayama et al., 2020 \\
\hline \multirow[t]{3}{*}{ BDE-209 } & Fish & Medaka (Oryzias latipes) & $96 \mathrm{~h}$ & $\mathrm{LC}_{50}$ & $>0.00455$ & MOEJ, 2012 \\
\hline & Crustacean & Water flea (Daphnia magna) & $48 \mathrm{~h}$ & $\mathrm{EC}_{50}$ & $>0.00479$ & MOEJ, 2012 \\
\hline & Algae & Green alga (Pseudokirchneriella subcapitata) & $72 \mathrm{~h}$ & $\mathrm{EC}_{50}$ & $>0.00520$ & MOEJ, 2012 \\
\hline \multirow[t]{3}{*}{ PBDMPP } & Fish & Rainbow trout (Oncorthynchus mykiss) & $96 \mathrm{~h}$ & $\mathrm{LC}_{50}$ & $>0.80$ & ECHA database \\
\hline & Crustacean & Water flea (Daphnia magna) & 21 days & NOEC & $>0.001$ & ECHA database \\
\hline & Algae & Freshwater algae & $72 \mathrm{~h}$ & $\mathrm{EC}_{50}$ & $>0.80$ & ECHA database \\
\hline PBDPP & Crustacean & Water flea (Daphnia magna) & $48 \mathrm{~h}$ & $\mathrm{EC}_{50}$ & $>66$ & Waaijers et al., 2013 \\
\hline \multirow[t]{3}{*}{ BPA-BDPP } & Fish & Rainbow trout (Oncorthynchus mykiss) & $72 \mathrm{~h}$ & $\mathrm{LC}_{50}$ & 0.17 & ECHA database \\
\hline & Crustacean & Freshwater invertebrates & 21 days & NOEC & 0.022 & ECHA database \\
\hline & Algae & Freshwater algae & $72 \mathrm{~h}$ & $\mathrm{EC}_{50}$ & 0.49 & ECHA database \\
\hline TDBP-TAZTO & Fish & Zebra fish (Danio rerio ) & 7 days & NOEC & 1 & Li et al., 2011 \\
\hline \multirow[t]{4}{*}{ DBDPE } & Fish & Zebra fish (Danio rerio ) & 8 days & NOEC & 0.0125 & Nakari and Huhtala, 2010 \\
\hline & Fish & Rainbow trout (Oncorthynchus mykiss) & $96 \mathrm{~h}$ & $\mathrm{LC}_{50}$ & $>110$ & Hardy et al., 2012 \\
\hline & Crustacean & Water flea (Daphnia magna) & $48 \mathrm{~h}$ & $\mathrm{EC}_{50}$ & $>110$ & Hardy et al., 2012 \\
\hline & Algae & Green algae (Pseudokirchneriella subcapitata) & $96 \mathrm{~h}$ & $\mathrm{EC}_{50}$ & $>110$ & Hardy et al., 2012 \\
\hline \multirow[t]{3}{*}{ TCIPP } & Fish & Common carp (Cyprinus carpio) & $96 \mathrm{~h}$ & $\mathrm{LC}_{50}$ & 31.9 & Bekele et al., 2018 \\
\hline & Crustacean & Water flea (Daphnia magna) & $48 \mathrm{~h}$ & $\mathrm{EC}_{50}$ & 91 & Verbruggen et al., 2005 \\
\hline & Algae & Green algae (Scenedesmus subspicatus) & $72 \mathrm{~h}$ & $\mathrm{EC}_{50}$ & 45 & Verbruggen et al., 2005 \\
\hline \multirow[t]{3}{*}{ TBOEP } & Fish & Rainbow trout (Oncorthynchus mykiss) & $96 \mathrm{~h}$ & $\mathrm{LC}_{50}$ & 24 & ECHA database \\
\hline & Crustacean & Water flea (Daphnia magna) & $48 \mathrm{~h}$ & $\mathrm{EC}_{50}$ & 53 & ECHA database \\
\hline & Algae & Green algae (Pseudokirchneriella subcapitata & $72 \mathrm{~h}$ & $\mathrm{EC}_{50}$ & 61 & ECHA datebase \\
\hline
\end{tabular}

${ }^{\text {a }}$ Toxicity was evaluated after 28 days. NOEC: no observed effect concentration, $\mathrm{LC}_{50}$ : lethal concentration $50 \%$, EC 50 : effective concentration $50 \%$. 
Table S15 Predicted no effect concentrations (PNECs) of 2,3,7,8-TCDD and FRs obtained for environmental risk assessment

\begin{tabular}{|c|c|c|c|c|c|c|c|c|}
\hline \multirow[b]{2}{*}{ Compound } & \multirow[b]{2}{*}{ Species } & \multirow{2}{*}{$\begin{array}{l}\text { Exposure } \\
\text { duration }\end{array}$} & \multirow[b]{2}{*}{ Criterion } & \multirow{2}{*}{$\begin{array}{l}\text { Value } \\
\text { (mg/L) }\end{array}$} & \multicolumn{3}{|c|}{ Assessment factor ${ }^{\mathrm{b}}$} & \multirow{2}{*}{$\begin{array}{l}\text { PNEC } \\
(\mathrm{ng} / \mathrm{L})\end{array}$} \\
\hline & & & & & $\begin{array}{c}\text { Interspecies } \\
\text { variation }\end{array}$ & ACR & LFE & \\
\hline 2,3,7,8-TCDD & Medaka (Oryzias latipes) & $6 \mathrm{~h}^{\mathrm{a}}$ & NOEC & 0.00000329 & 10 & - & 10 & 0.0329 \\
\hline BDE-209 & Medaka (Oryzias latipes) & $96 \mathrm{~h}$ & LC50 & $>0.00455$ & - & 100 & 10 & $>4.55$ \\
\hline PBDMPP & Water flea (Daphnia magna) & 21 days & NOEC & $>0.001$ & 10 & - & 10 & $>10$ \\
\hline PBDPP & Water flea (Daphnia magna) & $48 \mathrm{~h}$ & $\mathrm{EC}_{50}$ & $>66$ & 10 & 10 & 10 & $>66,000$ \\
\hline BPA-BDPP & Rainbow trout (Oncorthynchus mykiss) & 21 days & NOEC & 0.022 & 10 & - & 10 & 220 \\
\hline TDBP-TAZTO & Zebra fish (Danio rerio ) & 7 days & NOEC & 1 & 10 & - & 10 & 10,000 \\
\hline DBDPE & Zebra fish (Danio rerio ) & 8 days & NOEC & 0.0125 & 10 & - & 10 & 125 \\
\hline DBDPE & Rainbow trout (Oncorthynchus mykiss) & $96 \mathrm{~h}$ & $\mathrm{LC}_{50}$ & $>110$ & 10 & 100 & 10 & $>11,000$ \\
\hline TCIPP & Common carp (Cyprinus carpio ) & $96 \mathrm{~h}$ & $\mathrm{LC}_{50}$ & 31.9 & - & 100 & 10 & 31,900 \\
\hline TBOEP & Chinese rare minnow (Gobiocypris rarus) & $96 \mathrm{~h}$ & $\mathrm{LC}_{50}$ & 24 & - & 100 & 10 & $>24,000$ \\
\hline
\end{tabular}

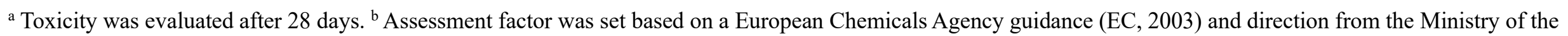
Environment of Japan (MOEJ, 2016). ACR: acute-to-chronic ratio; LFE, lab-to-field extrapolation. 


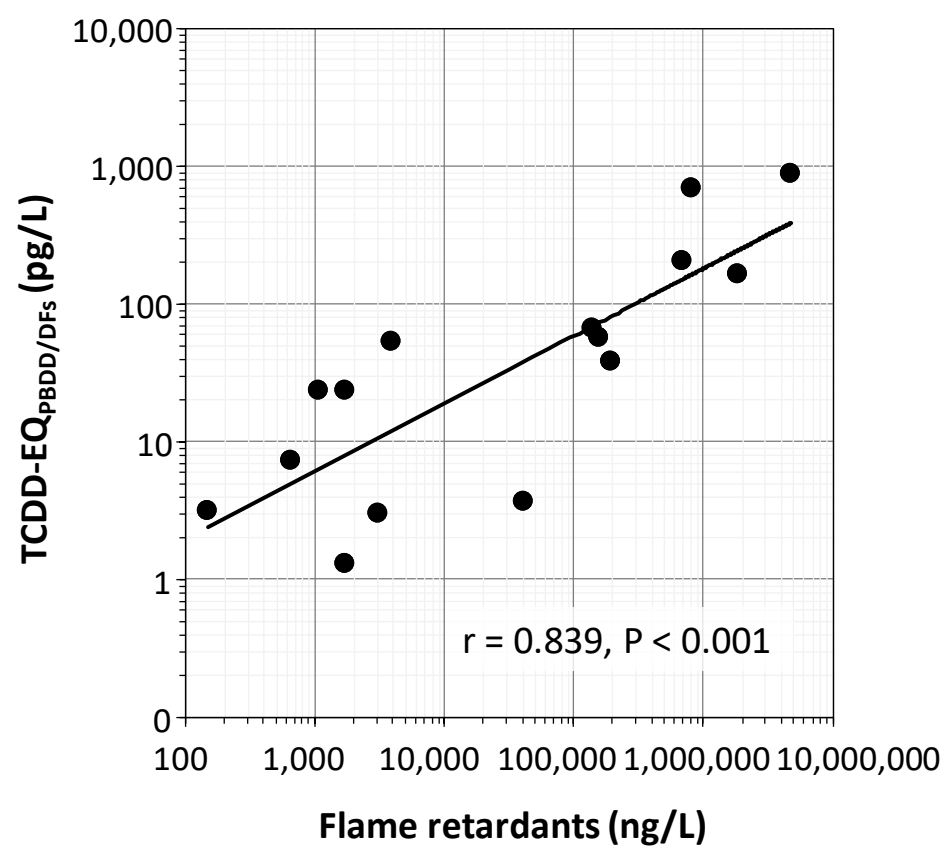

Figure S3. Correlation between TCDD-EQPBDD/DFs and flame retardants in the effluents of facilities handling decabromodiphenyl ether. Pearson correlation coefficient, $P$ value, and the values for individual samples are indicated.

TCDD-EQPBDD/DFs: TCDD equivalent for the brominated dioxin fraction. 